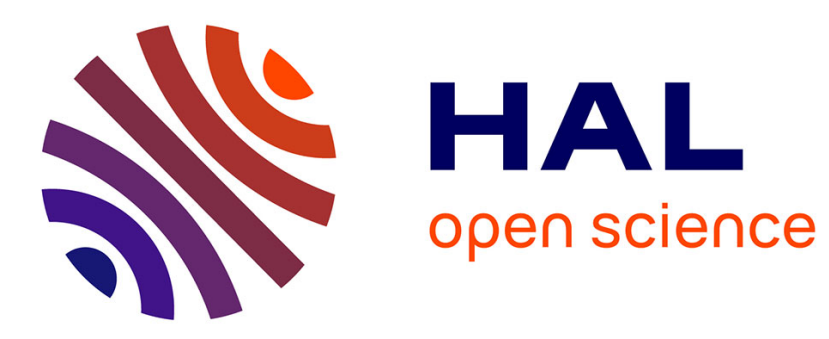

\title{
Parameter Synthesis for Bounded Cost Reachability in Time Petri Nets
}

Didier Lime, Olivier Henri Roux, Charlotte Seidner

\section{To cite this version:}

Didier Lime, Olivier Henri Roux, Charlotte Seidner. Parameter Synthesis for Bounded Cost Reachability in Time Petri Nets. 40th International Conference on Applications and Theory of Petri Nets and Concurrency (Petri Nets 2019), Jun 2019, Aachen, Germany. pp.406-425, 10.1007/978-3-03021571-2_22. hal-02565091

\section{HAL Id: hal-02565091 \\ https://hal.science/hal-02565091}

Submitted on 6 May 2020

HAL is a multi-disciplinary open access archive for the deposit and dissemination of scientific research documents, whether they are published or not. The documents may come from teaching and research institutions in France or abroad, or from public or private research centers.
L'archive ouverte pluridisciplinaire HAL, est destinée au dépôt et à la diffusion de documents scientifiques de niveau recherche, publiés ou non, émanant des établissements d'enseignement et de recherche français ou étrangers, des laboratoires publics ou privés. 


\title{
Parameter Synthesis for Bounded Cost Reachability in Time Petri Nets ${ }^{\star}$
}

\author{
Didier Lime ${ }^{1}$, Olivier H. Roux ${ }^{1}$, and Charlotte Seidner ${ }^{2}$ \\ ${ }^{1}$ École Centrale de Nantes, LS2N UMR CNRS 6004, France \\ ${ }^{2}$ Université de Nantes, LS2N UMR CNRS 6004, France
}

\begin{abstract}
We investigate the problem of parameter synthesis for time Petri nets with a cost variable that evolves both continuously with time, and discretely when firing transitions. More precisely, parameters are rational symbolic constants used for time constraints on the firing of transitions and we want to synthesise all their values such that the cost variable stays within a given budget.

We first prove that the mere existence of such values for the parameters is undecidable. We nonetheless provide a symbolic semi-algorithm that is proved both sound and complete when it terminates. We also show how to modify it for the case when parameters values are integers. Finally, we prove that this modified version terminates if parameters are bounded. While this is to be expected since there are now only a finite number of possible parameter values, this is interesting because the computation is symbolic and thus avoids an explicit enumeration of all those values. Furthermore, the result is a symbolic constraint representing a finite union of convex polyhedra that is easily amenable to further analysis through linear programming.

We finally report on the implementation of the approach in Romeo, a software tool for the analysis of hybrid extensions of time Petri nets.
\end{abstract}

\section{Introduction}

So-called priced or cost timed models are suitable for representing real-time systems whose behaviour is constrained by some resource consuming (be it energy or CPU time, for instance) and for which we need to assess the total cost accumulated during their execution. Such models can even describe whether the evolution of the cost during the run is caused by staying in a given state (continuous cost) or by performing a given action (discrete cost). Thus, the task of finding if the model can reach some "good" states while keeping the overall cost under a given bound (or, further, finding the minimum cost) can prove of interest in many real-life applications, such as optimal scheduling or production line planning.

Timed models, however, require a thorough knowledge of the system for their analysis and are thus difficult to build in the early design stages, when the

* This work is partially supported by the ANR national research program PACS (ANR-14-CE28-0002) 
system is not fully identified. Even when all timing constraints are known, the whole design process must often be caried out afresh, whenever the environment changes. To obtain such valuable characteristics as flexibility and robustness, the designer may want to relax constraints on some specifications by allowing them a wider range of values. To this end, parametric reasoning is particularly relevant for timed models, since it allows designers to use parameters instead of definite timing values.

We therefore propose to tackle the definition and analysis of models that support both (linear) cost functions and timed parameters.

Related work Parametric timed automata (PTA) [3] extend timed automata [2] to overcome the limits of checking the correctness of the systems with respect to definite timing constraints. The reachability-emptiness problem, which tests whether there exists a parameter valuation such that the automaton has an accepting run, is fundamental to any verification process but is undecidable [3]. $\mathrm{L} / \mathrm{U}$ automata [13] use each parameter either as a lower bound or as an upper bound on clocks. The reachability-emptiness problem is decidable for this model, but the state-space exploration, which would allow for explicit synthesis of all the suitable parameter valuations, still might not terminate [15]. To obtain decidability results, the approach described in [15] does not rely on syntactical restrictions on guards and invariants, but rather on restricting the parameter values to bounded integers. From a practical point of view, this subclass of PTA is not that restrictive, since the time constraints of timed automata are usually expressed as natural (or perhaps rational) numbers.

In [4], the authors have proved the decidability of the optimal-cost problem for Priced Timed Automata with non-negative costs. In $[7,8,16]$, the computation of the optimal-cost to reach a goal location is based on a forward exploration of zones extended with linear cost functions. In [12], the authors have improved this approach, so as to ensure termination of the forward exploration algorithm, even when clocks are not bounded and costs are negative, provided that the automaton has no negative cost cycles. In [1], the considered model is a timed arc Petri net, under weak firing semantics, extended with rate costs associated with places and firing costs associated with transitions. The computation of the optimal-cost for reaching a goal marking is based on similar techniques to [4]. In [11], the authors have investigated the optimal-cost reachability problem for time Petri nets where each transition has a firing cost and each marking has a rate cost (represented as a linear rate cost function over markings). To compute the optimal-cost to reach a goal marking, the authors have revisited the state class graph method to include costs.

Our contribution We propose in Section 2 an extension of time Petri nets with costs (both discrete and continuous with time) and timing parameters, i.e., rational symbolic constants used in the constraints on the firing times of transitions.

Within this formalism, we define two problems dealing with parametric reachability within a bounded cost. We prove in Section 3 that the existence of a 
parameter valuation to reach a given marking under a given bounded cost is undecidable. This proof adapts a 2-counter machine encoding first proposed in [14] for PTA. To our knowledge it is the first time a direct Petri net encoding is provided and the adaptation is not trivial. We give in Section 4 a symbolic semi-algorithm that computes all such parameter valuations when it terminates, and we prove its correctness. We propose in Section 5 a variant of this semialgorithm that computes integer parameter valuations and prove in Section 6 its termination provided those parameter valuations are bounded and the cost of each run is uniformly lower-bounded for integer parameter valuations. This technique is symbolic and avoids the explicit enumeration of all possible parameter valuations. The basic underlying idea of using the integer hull operator was first investigated in [15] for PTA, but this is the first time that it is adapted and proved to work with state classes for time Petri nets, and the fact that it naturally also preserves costs for integer parameter valuations is new and very interesting. We finally describe in Section 7 the implementation of the approach in the tool Romeo by analysing a small scheduling case-study.

\section{Parametric Cost Time Petri Nets}

\subsection{Preliminaries}

We denote the set of natural numbers (including 0 ) by $\mathbb{N}$, the set of integers by $\mathbb{Z}$, the set of rational numbers by $\mathbb{Q}$ and the set of real numbers by $\mathbb{R}$. We note $\mathbb{Q}_{\geq 0}$ (resp. $\mathbb{R}_{\geq 0}$ ) the set of non-negative rational (resp. real) numbers. For $n \in \mathbb{N}$, we let $\llbracket 0, n \rrbracket$ denote the set $\{i \in \mathbb{N} \mid i \leq n\}$. For a finite set $X$, we denote its size by $|X|$.

Given a set $X$, we denote by $\mathcal{I}(X)$, the set of non empty real intervals that have their finite end-points in $X$. For $I \in \mathcal{I}(X), \underline{I}$ denotes its left end-point if $I$ is left-bounded and $-\infty$ otherwise. Similarly, $\bar{I}$ denotes the right end-point if $I$ is right-bounded and $\infty$ otherwise. We say that an interval $I$ is non-negative if $I \subseteq \mathbb{R}_{\geq 0}$. Moreover, for any $d \in \mathbb{R}_{\geq 0}$ and any non-negative interval $I$, we let $I \ominus d$ be the interval defined by $\{\theta-d \mid \theta \in I \wedge \theta-d \geq 0\}$. Note that this is again a non-negative interval.

Given sets $V$ and $X$, a $V$-valuation (or simply valuation when $V$ is clear from the context) of $X$ is a mapping from $X$ to $V$. We denote by $V^{X}$ the set of $V$-valuations of $X$. When $X$ is finite, given an arbitrary fixed order on $X$, we often equivalently consider $V$-valuations as vectors of $V^{|X|}$. Given a $V$-valuation $v$ of $X$ and $Y \subseteq X$, we denote by $v_{\mid Y}$ the projection of $v$ on $Y$, i.e., the valuation on $Y$ such that $\forall x \in Y, v_{\mid Y}(x)=v(x)$.

\subsection{Time Petri Nets with Costs and Parameters}

Definition 1 (Parametric Cost Time Petri Net (pcTPN)). A Parametric Cost Time Petri Net $(p c T P N)$ is a tuple $\mathcal{N}=\left(P, T, \mathbb{P}, \cdot ., \bullet^{\bullet}, m_{0}, I_{s}\right.$, cost $_{t}$, cost $\left._{m}\right)$ where 
- $P$ is a finite non-empty set of places,

$-T$ is a finite set of transitions such that $T \cap P=\emptyset$,

- $\mathbb{P}$ is a finite set of parameters,

-.$: T \rightarrow \mathbb{N}^{P}$ is the backward incidence mapping,

$-\because: T \rightarrow \mathbb{N}^{P}$ is the forward incidence mapping,

$-m_{0} \in \mathbb{N}^{P}$ is the initial marking,

- $I_{s}: T \rightarrow \mathcal{I}(\mathbb{N} \cup \mathbb{P})$ is the (parametric) static firing interval function,

- cost $_{t}: T \rightarrow \mathbb{Z}$ is the discrete cost function, and

- cost $_{m}: \mathbb{N}^{P} \rightarrow \mathbb{Z}$ is the cost rate function.

Given a parameterized object $x$ (be it a pcTPN, a function, an expression, etc.), and a $\mathbb{Q}$-valuation $v$ of parameters, we denote by $v(x)$ the corresponding non-parameterized object, in which each parameter $a$ has been replaced by the value $v(a)$.

A marking is an $\mathbb{N}$-valuation of $P$. For a marking $m \in \mathbb{N}^{P}, m(p)$ represents a number of tokens in place $p$. A transition $t \in T$ is said to be enabled by a given marking $m \in \mathbb{N}^{P}$ if for all places $p, m(p) \geq{ }^{\bullet} t(p)$. We also write $m \geq{ }^{\bullet}$. We denote by en $(m)$ the set of transitions that are enabled by the marking $m$ : en $(m)=\{t \in T \mid m \geq \bullet\}$.

Firing an enabled transition $t$ from marking $m$ leads to a new marking $m^{\prime}=$ $m-{ }^{\bullet} t+t^{\bullet}$. A transition $t^{\prime} \in T$ is said to be newly enabled by the firing of a transition $t$ from a given marking $m \in \mathbb{N}^{P}$ if it is enabled by the new marking but not by $m-\bullet$ (or it is itself fired). We denote by newen $(m, t)$ the set of transitions that are newly enabled by the firing of $t$ from the marking $m$ : newen $(m, t)=$ $\left\{t^{\prime} \in \operatorname{en}\left(m-\bullet t+t^{\bullet}\right) \mid t^{\prime} \notin\right.$ en $(m-\bullet t)$ or $\left.t=t^{\prime}\right\}$

A state of the net $\mathcal{N}$ is a tuple $(m, I, c, v)$ in $\mathbb{N}^{P} \times \mathcal{I}\left(\mathbb{R}_{\geq 0}\right)^{T} \times \mathbb{R} \times \mathbb{Q}_{\geq 0}^{\mathbb{P}}$, where: $m$ is a marking of $\mathcal{N}, I$ is called the interval function and associates a temporal interval to each transition enabled by $m$. Value $c$ is the cost associated with that state and valuation $v$ assigns a rational value to each parameter for the state.

Definition 2 (Semantics of a pcTPN). The semantics of a pcTPN is a timed transition system $\left(Q, Q_{0}, \rightarrow\right)$ where:

- $Q \subseteq \mathbb{N}^{P} \times \mathcal{I}\left(\mathbb{R}_{\geq 0}\right)^{T} \times \mathbb{R} \times \mathbb{Q}_{\geq 0}^{\mathbb{P}}$

- $Q_{0}=\left\{\left(m_{0}, I_{0}, 0, v\right) \mid v \in \mathbb{Q}_{\geq 0}^{\mathbb{P}}, \forall t \in T, v\left(I_{s}(t)\right) \neq \emptyset\right\}$ where $\forall t \in$ en $\left(m_{0}\right), I_{0}(t)=$ $I_{s}(t)$

$-\rightarrow$ consists of two types of transitions:

- discrete transitions: $(m, I, c, v) \stackrel{t \in T}{\longrightarrow}\left(m^{\prime}, I^{\prime}, c^{\prime}, v\right)$ iff

$* m \geq \bullet, m^{\prime}=m-\bullet t+t^{\bullet}$ and $v(I(t))=0$,

$* \forall t^{\prime} \in$ en $\left(m^{\prime}\right)$

- $I^{\prime}\left(t^{\prime}\right)=I_{s}\left(t^{\prime}\right)$ if $t^{\prime} \in \operatorname{newen}(m, t)$,

- $I^{\prime}\left(t^{\prime}\right)=I\left(t^{\prime}\right)$ otherwise

$* c^{\prime}=c+\operatorname{cost}_{t}(t)$

- time transitions: $(m, I, c, v) \stackrel{d \in \mathbb{R}_{\geq 0}}{\longrightarrow}\left(m, I \ominus d, c^{\prime}, v\right)$, iff $\forall t \in$ en $(m)$, $\overline{(I \ominus d)(t)} \geq 0$ and $c^{\prime}=c+\operatorname{cost}_{m}(m) * d$. 
A run of a pcTPN $\mathcal{N}$ is a (finite or infinite) sequence $q_{0} a_{0} q_{1} a_{1} q_{2} a_{2} \cdots$ such that $q_{0} \in Q_{0}$, for all $i>0, q_{i} \in Q, a_{i} \in T \cup \mathbb{R}_{\geq 0}$ and $q_{i} \stackrel{a_{i}}{\longrightarrow} q_{i+1}$. The set of runs of $\mathcal{N}$ is denoted by $\operatorname{Runs}(\mathcal{N})$. We note $(m, I, c, v) \stackrel{t @ d}{\longrightarrow}\left(m^{\prime}, I^{\prime}, c^{\prime}, v\right)$ for the sequence of elapsing $d \geq 0$ followed by the firing of the transition $t$. We denote by sequence $(\rho)$ the projection of the run $\rho$ over $T$ : for a run $\rho=q_{0} \stackrel{t_{0} @ d_{0}}{\longrightarrow} q_{1} \stackrel{t_{1} @ d_{1}}{\longrightarrow}$ $q_{2} \stackrel{t_{2} @ d_{2}}{\longrightarrow} q_{3} \stackrel{t_{3} @ d_{3}}{\longrightarrow} \cdots$, we have sequence $(\rho)=t_{0} t_{1} t_{2} t_{3} \cdots$. We write $q \stackrel{t}{\hookrightarrow} q^{\prime}$ if there exists $d \geq 0$ such that $q \stackrel{t @ d}{\longrightarrow} q^{\prime}$.

For a finite run $\rho$ we denote by last $(\rho)$ the last state of $\rho$ and by lastm $(\rho)$ its marking. A state $(m, I, c, v)$ is said to be reachable if there exists a finite run $\rho$ of the net, with last $(\rho)=(m, I, c, v)$. A marking $m$ is reachable for parameter valuation $v$, if there exists some $I$ and $c$ such that $(m, I, c, v)$ is reachable.

For $k \in \mathbb{N}$ and parameter valuation $v$, the (Cost) Time Petri net $v(\mathcal{N})$ is said to be $k$-bounded if for all reachable markings $m$, and all places $p, m(p) \leq k$. We say that $v(\mathcal{N})$ is bounded if there exists $k$ such that it is $k$-bounded.

The cost $\operatorname{cost}(\rho)$ of a finite run $\rho$, with last state $(m, I, c, v)$ is $c$. Since we are interested in minimising the cost, the cost of a sequence of transitions $\sigma$ is defined as $\operatorname{cost}(\sigma)=\inf _{\rho \in \operatorname{Runs}(\mathcal{N}) \text {, sequence }(\rho)=\sigma} \operatorname{cost}(\rho)$. For the sake of the clarity of the presentation, we consider only closed intervals (or right-open to infinity) so this infimum is actually a minimum.

\subsection{Parametric Cost Problems}

Given a set of target markings Goal, the problems we are interested in are:

1. the existential problem: Given a finite maximum cost value $c_{\max }$, is there a parameter valuation $v$ such that some marking in Goal is reachable with a cost less than $c_{\max }$ in $v(\mathcal{N})$ ?

2. the synthesis problem: Given a finite maximum cost value $c_{\max }$, compute all the parameter valuations $v$ such that some marking in Goal is reachable with a cost less than $c_{\max }$ in $v(\mathcal{N})$.

We prove in Section 3 that the existential problem is undecidable.

\section{$3 \quad$ Undecidability Results}

The existential parametric time bounded reachability problem for bounded parametric time Petri nets asks whether a given target marking is reachable for some valuation of the parameter(s) within $c_{\max }$ time units. This is a special case of the existential cost bounded reachability problem defined in Section 2, with no discrete cost and a uniform cost rate of 1 . Proposition 1 therefore implies the undecidability of that more general problem.

Proposition 1. Existential parametric time bounded reachability is undecidable for bounded parametric time Petri nets. 


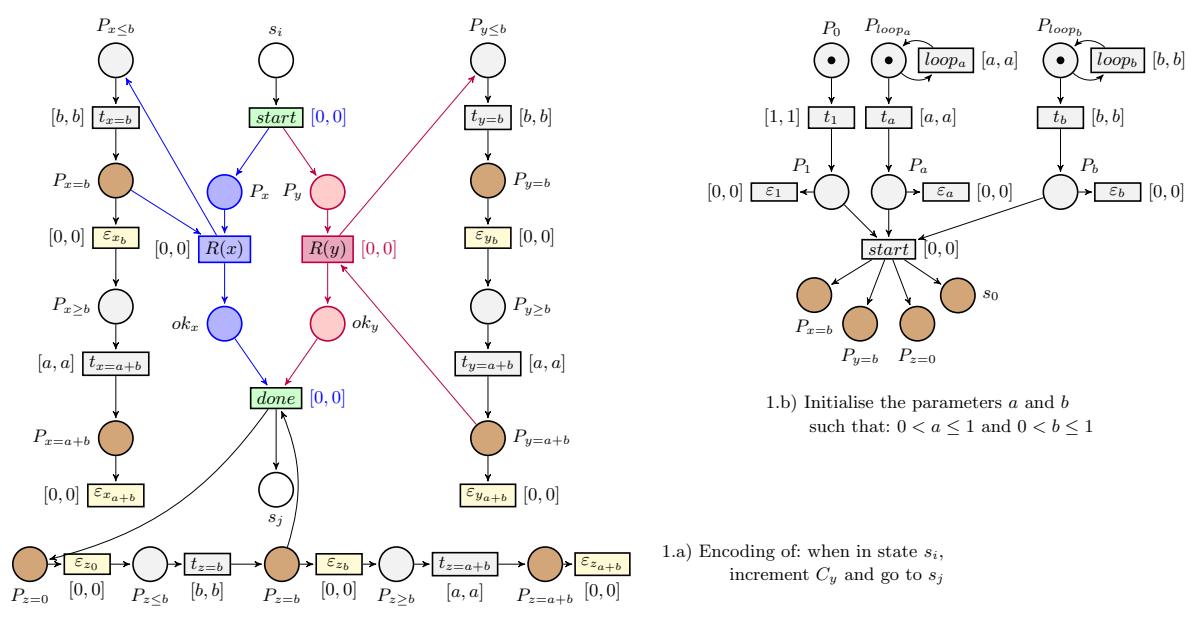

Fig. 1. Increment Gadget (left) and Initial gadget (right)

Proof. Given a bounded parametric time Petri net $\mathcal{N}$, we want to decide whether there exists some parameter valuation $v$ such that some given marking can be reached within $c_{\max }$ time units in $v(\mathcal{N})$. The idea of this proof was first sketched in [14] for parametric timed automata. We encode the halting problem for twocounter machines, which is undecidable [18], into the existential problem for parametric timed Petri nets. Recall that a 2-counter machine $\mathcal{M}$ has two nonnegative counters (here $C_{x}$ and $C_{y}$ ), a finite number of states and a finite number of transitions, which can be of the form: 1 ) when in state $s_{i}$, increment a counter and go to $\left.s_{j} ; 2\right)$ when in state $s_{i}$, decrement a counter and go to $\left.s_{j} ; 3\right)$ when in state $s_{i}$, if a counter is null then go to $s_{j}$, otherwise block. The machine starts in state $s_{0}$ and halts when it reaches a particular state $s_{\text {halt }}$.

Given such a machine $\mathcal{M}$, we now provide an encoding as a parametric time Petri net $\mathcal{N}_{\mathcal{M}}$ : each state $s_{i}$ of the machine is encoded as place, which we also call $s_{i}$. The encoding of the 2 -counter machine $\mathcal{M}$ is as follows: it uses two rationalvalued parameter $a$ and $b$, and three gadgets shown in Figure 1.a modelling three clocks $x, y, z$. Recall that, for a state $(m, I, c, v)$, the enabling time of an enabled transition $t$ is $v\left(\overline{I_{s}(t)}-\overline{I(t)}\right)$. For the gadget modelling the clock $x$, the value of the clock $x$ is equal to: i) the enabling time of the transition $t_{x=b}$ when $P_{x \leq b}$ is marked; ii) $b$ when $P_{x \leq b}$ is marked; iii) the sum of $b$ and the enabling time of the transition $t_{x=a+b}$ when $P_{x \geq b}$ is marked (note that this value is lower than $a+b)$; iv) $a+b$ when $P_{x=a+b}$ is marked; v) an unknown (an irrelevant) value in all other cases.

The gadget encoding the increment instruction of $C_{y}$ is given in Figure 1.a. The clocks $x$ and $y$ store the value of each counter $C_{x}$ and $C_{y}$ as follows $x=$ $b-a . C_{x}$ and $y=b-a . C_{y}$ when $z=0$. The zero-test gadget is given in Figure 2 . We use the initial gadget in Figure 1.b to initialise $a$ and $b$ such that $0<a \leq 1$ and $0<b \leq 1$. The system is studied over 1 time unit. 
Increment: We start from some encoding configuration: $x=b-a . C_{x}, y=$ $b-a . C_{y}$ and $z=0$ in a marking such that the places $P_{z=0}$ and $s_{i}$ are marked. After the firing of the transition start, there is an interleaving of the transitions $R(x)$ and $R(y)$ that go through the gadget. Finally, we can fire the transition done when $z=b$ (i.e. $b-a . C_{x}$ later) and we have $z=0, x=b-a . C_{x}$ and $y=b-a\left(C_{y}+1\right)$ as expected. Moreover, $v\left(\mathcal{N}_{\mathcal{M}}\right)$ will block for all the parameter valuations $v$ which not correctly encode the machine.

Decrement: By replacing the arc from $P_{z=b}$ to done by an arc from $P_{z=a+b}$ to done, the only difference in the previous reasoning is that the elapsing time to fire done is increased of $a$. Then we obtain $z=0, x=b+a-a . C_{x}=b-a \cdot\left(C_{x}-1\right)$ and $y=b-a . C_{y}$ corresponding to the decrement of $C_{x}$.

We can obtain symmetrically (by swapping $x$ and $y$ ) the increment of $C_{x}$ and the decrement of $C_{y}$

Both the increment gadget and the zero-test gadget require $b$ time units, and the decrement gadget requires $(a+b)$ time units. Since the system executes over 1 time unit, for any value of $a>0$ and $b>0$, the number of operations that the machine can perform is finite. We consider two cases:

1. Either the machine halts, both counters $C_{x}$ and $C_{y}$ are bounded (let $c$ their maximum value) and the halting and finite execution of the machine is within $m$ steps. If $c=0$ then the machine is a sequence of $m$ zero-test taking $m . b$ time units and the parametric Petri net $\mathcal{N}_{\mathcal{M}}$ can go within 1 time unit to a marking $m_{\text {halt }}$ if $0<a \leq 1$ and $0<b \leq \frac{1}{m}$. If $c>0$, since an instruction requires at most $a+b$ time units, if $a+b \leq \frac{1}{m}$ and if $0<a \leq \frac{b}{c}$ then there exists a run that correctly simulates the machine, and eventually reaches $m_{\text {halt }}$ within 1 time unit.

This set of valuations is non-empty: for example if $c=0$, then we can choose $a=b=\frac{1}{m}$ and if $c>0$, then, since $m \geq c$, we can choose $a=\frac{b}{m}$ and $b=\frac{1}{1+m}$ hence $a=\frac{1}{m(1+m)}$.

2. Or the machine does not halt. A step requires at least $b$ time units then for any value $v$ of the parameters, after a maximum number of steps (at most $\left.\frac{1}{b}\right)$, one whole time unit will elapse without $v\left(\mathcal{N}_{\mathcal{M}}\right)$ reaching $m_{\text {halt }}$.

\section{A Symbolic Semi-algorithm for Parameter Synthesis}

\subsection{State Classes}

We now introduce the notion of state classes for pcTPNs. It was originally introduced for time Petri nets in [10,9], and extended for timing parameters in [21], and for costs in [11]. We show that those two extensions seamlessly blend together.

For an arbitrary sequence of transitions $\sigma=t_{1} \ldots t_{n} \in T^{*}$, let $C_{\sigma}$ be the set of all states that can be reached by the sequence $\sigma$ from any initial state $q_{0}$ :

$C_{\sigma}=\left\{q \in Q \mid q_{0} \stackrel{t_{1}}{\longrightarrow} q_{1} \ldots \stackrel{t_{n}}{\longrightarrow} q\right\}$. All the states of $C_{\sigma}$ share the same marking and can therefore be written as a pair $(m, D)$ where $m$ is the common marking 


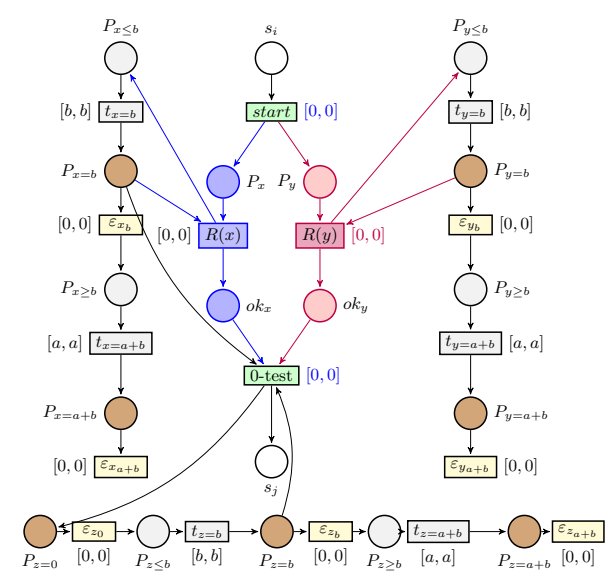

Fig. 2. Encoding 0-test over bounded-time: when in state $s_{i}$, if $C_{x}=0$ then go to $s_{j}$

and, if we note en $(m)=\left\{t_{1}, \ldots, t_{n}\right\}$, then $D$ is the set of points $\left(\theta_{1}, \ldots, \theta_{n}, c, v\right)$ such that $(m, I, c, v) \in C_{\sigma}$ and for all $t_{i} \in \operatorname{en}(m), \theta_{i} \in I\left(t_{i}\right)$. For short, we will often write $(\vec{\theta}, c, v)$ for such a point, with $\vec{\theta}=\left(\theta_{1}, \ldots, \theta_{n}\right)$ and a small abuse of notation. We denote by $\Theta$ the set of $\theta_{i}$ variables, of which we have one per transition of the net: for the sake of simplicity, we will usually use the same index to denote for instance that $\theta_{i}$ corresponds to transition $t_{i}$.

$C_{\sigma}$ is called a state class and $D$ is its firing domain.

Lemma 1 equivalently characterises state classes, as a straigthforward reformulation of the definition:

Lemma 1. For all classes $C_{\sigma}=(m, D),(\vec{\theta}, c, v) \in D$ if and only if there exists a run $\rho$ in $v(\mathcal{N})$, and $I:$ en $(m) \rightarrow \mathcal{I}\left(\mathbb{Q}_{\geq 0}\right)$, such that sequence $(\rho)=\sigma,(m, I, c)=$ last $(\rho)$, and $\vec{\theta} \in I$.

From Lemma 1, we can then deduce a characterisation of the "next" class, obtained by firing a firable transition from some other class. This is expressed by Lemma 2 .

Lemma 2. Let $C_{\sigma}=(m, D)$ and $C_{\sigma . t_{f}}=\left(m^{\prime}, D^{\prime}\right)$, we have:

$$
\left(\overrightarrow{\theta^{\prime}}, c^{\prime}, v\right) \in D^{\prime} \text { iff } \exists(\vec{\theta}, c, v) \in D \text { s.t. }\left\{\begin{array}{l}
\forall t_{i} \in \operatorname{en}(m), \theta_{i}-\theta_{f} \geq 0 \\
\forall t_{i} \in \operatorname{en}\left(m-t_{f}\right), \theta_{i}^{\prime}=\theta_{i}-\theta_{f} \\
\forall t_{i} \in \operatorname{newen}\left(m, t_{f}\right), \theta_{i}^{\prime} \in v\left(I_{s}\right)\left(t_{i}\right) \\
c^{\prime}=c+\operatorname{cost}_{m}(m) * \theta_{f}+\operatorname{cost}_{t}\left(t_{f}\right)
\end{array}\right.
$$

Proof. Consider $\left(\vec{\theta}^{\prime}, c^{\prime}, v\right) \in D^{\prime}$. Then by Lemma 1, there exists a run $\rho^{\prime}$ in $v(\mathcal{N})$, and $I^{\prime}: \operatorname{en}(m) \rightarrow \mathcal{I}\left(\mathbb{Q}_{\geq 0}\right)$, such that sequence $\left(\rho^{\prime}\right)=\sigma . t_{f},\left(m^{\prime}, I^{\prime}, c^{\prime}\right)=\operatorname{last}\left(\rho^{\prime}\right)$, and $\overrightarrow{\theta^{\prime}} \in I^{\prime}$. Consider the prefix $\rho$ of $\rho^{\prime}$ such that sequence $(\rho)=\sigma$. The last state of $\rho$ can be written $(m, I, c, v)$ for some $I$ and $c$. We know that $t_{f}$ is fired from 
$(m, I, c, v)$ so there exists some delay $d$ such that $I\left(t_{f}\right) \leq d$ and for all other transitions $t_{i}$ enabled by $m, \overline{I\left(t_{i}\right)} \geq d$. Furthermore, $c=c^{\prime}-\operatorname{cost}_{m}(m) * d-$ $\operatorname{cost}_{t}\left(t_{f}\right)$. It follows that there exists a point $\vec{\theta} \in I$ with the desired properties.

The other direction is similar.

Note that according to Lemma $2, D^{\prime}$ is not empty if and only if there exists $(\vec{\theta}, c, v)$ in $D$ such that for all $t_{i} \in$ en $(m), \theta_{i} \geq \theta_{f}$. In that case we say that $t_{f}$ is firable from $(m, D)$ and note $t_{f} \in$ firable $((m, D))$.

From Lemma 2, it follows that $C_{\sigma . t_{f}}$ can be computed from $C_{\sigma}$ using Algorithm 1 . Note that it is formally the same algorithm as in [11].

Given a class $C$ and a transition $t$ firable from $C$, we note $\operatorname{Next}(C, t)$ the result of applying Algorithm 1 to $C$ and $t$.

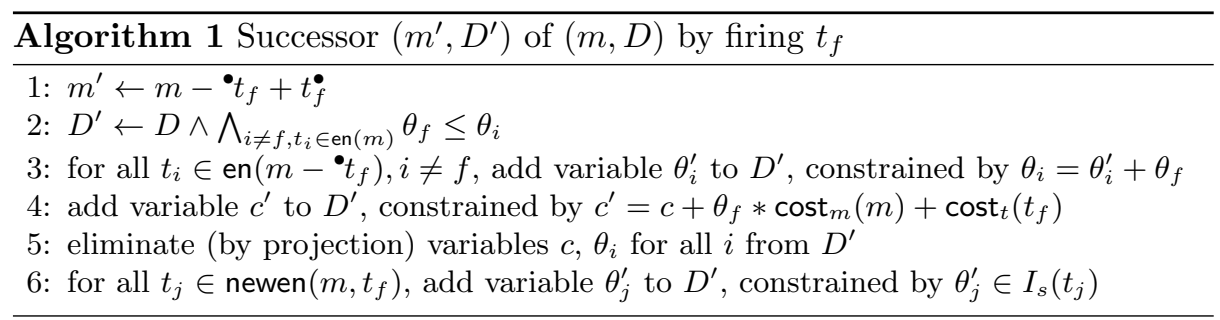

Let $C_{0}=\left(m_{0}, D_{0}\right)$ be the initial class. Domain $D_{0}$ is defined by the constraints $\forall t_{i} \in \operatorname{en}\left(m_{0}\right), \theta_{i} \in I_{s}\left(t_{i}\right), \forall t \in T, I_{s}(t) \neq \emptyset$, and $c=0$. This gives a convex polyhedron of $\mathbb{R}_{\geq 0}^{\mid \text {en }\left(m_{0}\right)|+| \mathbb{P} \mid+1}$; since all the operations on domains in Algorithm 1 are polyhedral, all the domains of state classes are also convex polyhedra. Note that only enabled transitions are constrained in the domain of a state class.

Naturally, we define the cost of state class $C_{\sigma}$ as $\operatorname{cost}\left(C_{\sigma}\right)=\operatorname{cost}(\sigma)$.

\subsection{The Synthesis Semi-algorithm}

In Algorithm 2, we explore the symbolic state-space in a classic manner. Whenever a goal marking is encountered we collect the parameter valuations that allowed that marking to be reached with a cost less or equal to $c_{\max }$.

The PASSED list records the visited symbolic states. Instead of checking new symbolic states for membership, we test a weaker relation denoted by $\preccurlyeq$ : does there exist a visited state allowing more behaviors with a cheaper cost?

For any state class $C=(m, D)$ and any point $(\vec{\theta}, v) \in D_{\mid \Theta \cup \mathbb{P}}$, the optimal cost of $(\vec{\theta}, v)$ in $D$ is defined by $\operatorname{cost}_{D}(\vec{\theta}, v)=\inf _{(\vec{\theta}, c, v) \in D} c$.

Definition 3. Let $C=(m, D)$ and $C^{\prime}=\left(m^{\prime}, D^{\prime}\right)$ be two parametric cost state classes. We say that $C$ is subsumed by $C^{\prime}$, which we denote by $C \preccurlyeq C^{\prime}$ iff $m=m^{\prime}, D_{\mid \Theta \cup \mathbb{P}} \subseteq D_{\mid \Theta \cup \mathbb{P}}^{\prime}$, and for all $(\vec{\theta}, v) \in D_{\mid \Theta \cup \mathbb{P}}, \operatorname{cost}_{D^{\prime}}(\vec{\theta}, v) \leq \operatorname{cost}_{D}(\vec{\theta}, v)$. 


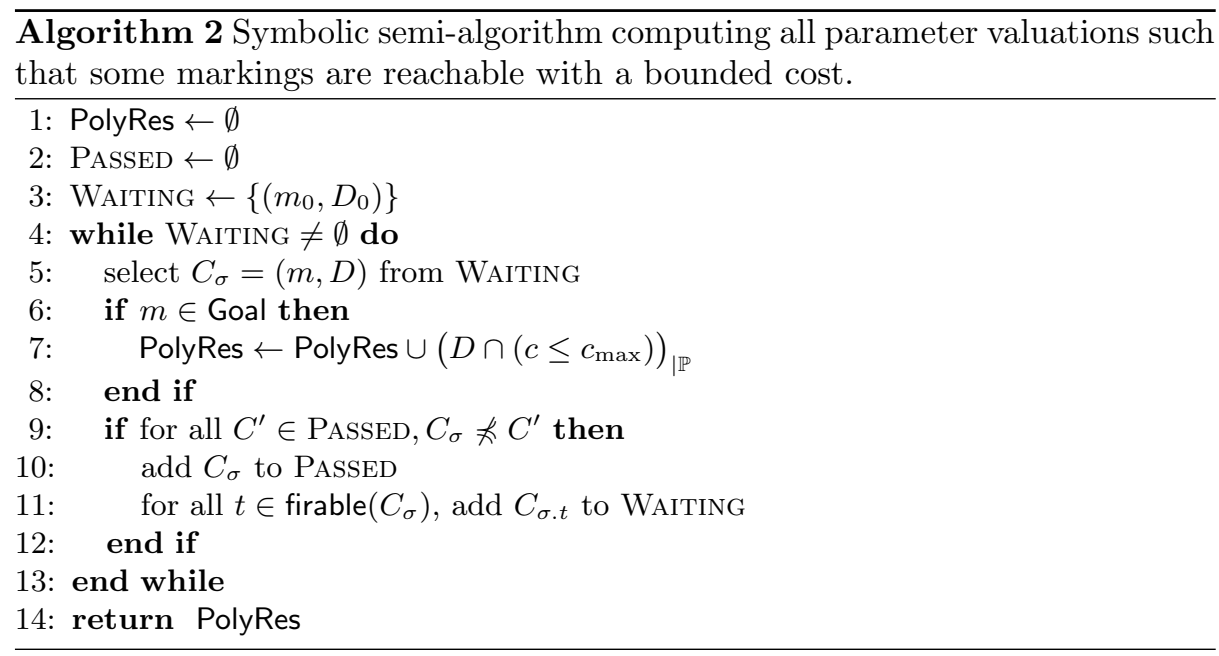

The following result is a fairly direct consequence of Definition 3 :

Lemma 3. Let $C_{\sigma_{1}}$ and $C_{\sigma_{2}}$ be two state classes such that $C_{\sigma_{1}} \preccurlyeq C_{\sigma_{2}}$.

If a transition sequence $\sigma$ is firable from $C_{\sigma_{1}}$, it is also firable from $C_{\sigma_{2}}$ and $\operatorname{cost}\left(C_{\sigma_{1} . \sigma}\right) \geq \operatorname{cost}\left(C_{\sigma_{2} . \sigma}\right)$.

Proof. Let $C_{\sigma_{1}}=\left(m_{1}, D_{1}\right)$ and $C_{\sigma_{2}}=\left(m_{2}, D_{2}\right)$. From Definition 3, for any point $\left(\vec{\theta}, c_{1}, v\right) \in D_{1}$, there exists a point $\left.\left(\vec{\theta}, c_{2}, v\right) \in D_{2}\right)$ such that $c_{2} \leq c_{1}$. This implies that: (i) $\operatorname{cost}\left(C_{\sigma_{1}}\right) \geq \operatorname{cost}\left(C_{\sigma_{2}}\right)$; (ii) if transition $t$ is firable from $C_{\sigma_{1}}$, then it is firable from $C_{\sigma_{2}}$ and $\operatorname{Next}\left(C_{\sigma_{1}}, t\right) \preccurlyeq \operatorname{Next}\left(C_{\sigma_{2}}, t\right)$. And the result follows by a straightforward induction.

While $\preccurlyeq$ can be checked using standard linear algebra techniques, we can also reduce it to standard inclusion on polyhedra by removing the upper bounds on cost (an operation called cost relaxation) [11].

Lemma 4. The following invariant holds after each iteration of the while loop in Algorithm 2: for all $C_{\sigma}=(m, D) \in$ PASSED,

1. for all prefixes $\sigma^{\prime}$ of $\sigma, C_{\sigma^{\prime}} \in$ PASSED;

2. if $m \in$ Goal then $\left(D \cap\left(c \leq c_{\max }\right)\right)_{\mid \mathbb{P}} \subseteq$ PolyRes;

3. if $t$ is firable from $C_{\sigma}$

- either $C_{\sigma . t} \in$ WAITING,

- or there exists $C^{\prime} \in$ PASSED such that $C_{\sigma . t} \preccurlyeq C^{\prime}$.

Proof. We prove this lemma by induction. Before the while loop starts, PASSED is empty so the invariant is true. Let us now assume that the invariant holds for all iterations up to the $n$-th one, with $n \geq 0$, and that WAITING $\neq \emptyset$. Let $C_{\sigma} \in$ WAIting be the selected class at line 5 ; to check whether the invariant still holds at the end of the $(n+1)$-th iteration, we only have to test the case where $C_{\sigma}$ is added to PASSED (which means that the condition at line 9 is true). We can then check each part of the invariant: 
1. $C_{\sigma}$ was picked from Waiting (line 5); except for the initial class (for which $\sigma$ is empty, and therefore has no prefix), it means that, in a previous iteration, there was a sequence $\sigma^{\prime}$ and a transition $t \in$ firable $\left(C_{\sigma^{\prime}}\right)$ such that $\sigma=\sigma^{\prime} . t$ (line 11) and $C_{\sigma^{\prime}} \in$ PAssed (line 10). Since we add at most one state class to PASSED at each iteration, $C_{\sigma^{\prime}}$ was added in a previous iteration and we can apply to it the induction hypothesis, which allows us to prove the first part of the invariant;

2. lines 6 and 7 obviously imply the second part of the invariant;

3. if $C_{\sigma} \in$ PASSED, then the condition of the if on line 9 is true and then for any transition $t$ that is firable from $C_{\sigma}, C_{\sigma . t}$ is added to WAIting (line 11) so the third part of the invariant holds for $C_{\sigma}$. Nevertheless, $C_{\sigma}$ itself is no longer in WAITING, and it is (except for the initial state class) the successor of some state class in PASSED. But then we have only two possibilities: either $C_{\sigma}$ has been added to PASSED in line 10 if the condition on line 9 was true, and certainly $C_{\sigma} \preccurlyeq C_{\sigma}$, or there exists $C^{\prime} \in$ PASSED such that $C_{\sigma} \preccurlyeq C^{\prime}$ if that condition was false. Therefore the third part of the invariant holds.

Both the basis case and the induction step are true: the result follows by induction.

Proposition 2. After any iteration of the while loop in Algorithm 2:

1. if $v \in$ PolyRes, then there exists a run $\rho$ in $v(\mathcal{N})$ such that $\operatorname{cost}(\rho) \leq c_{\max }$ and $\operatorname{lastm}(\rho) \in$ Goal.

2. if WAITING $=\emptyset$ then, for all parameter valuations $v$ such that there exists a run $\rho$ in $v(\mathcal{N})$ such that $\operatorname{cost}(\rho) \leq c_{\max }$ and $\operatorname{lastm}(\rho) \in$ Goal, we have $v \in$ PolyRes.

Proof. 1. By induction on the while loop: initially, PolyRes is empty so the result holds trivially. Suppose it holds after some iteration $n$, and consider iteration $n+1$. Let $v \in$ PolyRes after iteration $n+1$. If $v$ was already in PolyRes after iteration $n$ then we can apply the induction hypothesis. Otherwise it means that if $C_{\sigma}=(m, D)$ is the class examined at iteration $n+1$, then $m \in$ Goal and $v \in\left(D \cap\left(c \leq c_{\max }\right)\right)_{\mid \mathbb{P}^{*}}$ This means that there exists some point $(\vec{\theta}, c, v) \in D$ with $c \leq c_{\max }$. By Lemma 1 , this means that there exists a run $\rho$ such that $(m, I, c, v)=\operatorname{last}(\rho)$, for some $I$ such that $\vec{\theta} \in I$, and therefore $\operatorname{lastm}(\rho) \in \mathrm{Goal}$ and $\operatorname{cost}(\rho) \leq c_{\max }$.

2. Let $v$ be a parameter valuation such that there exists a run $\rho$ in $v(\mathcal{N})$ such that $\operatorname{cost}(\rho) \leq c_{\max }$ and $\operatorname{lastm}(\rho) \in$ Goal. Let $\sigma=$ sequence $(\rho)$. We proceed by induction on the length $n$ of the biggest suffix $\sigma_{2}$ of $\sigma$ such that, either $\sigma_{2}$ is empty or, if we note $\sigma=\sigma_{1} \sigma_{2}$, with the first element of $\sigma_{2}$ being transition $t$, then $C_{\sigma_{1} t} \notin$ PASSED.

If $n=0$, then $C_{\sigma}=(m, D) \in$ PAssed. By Lemma $1, v \in D_{\mid \mathbb{P}}$ and $m \in$ Goal. From the latter, we have $\left(D \cap\left(c \leq c_{\max }\right)\right)_{\mid \mathbb{P}} \subseteq$ PolyRes and therefore $v \in$ PolyRes because $v \in\left(D \cap\left(c \leq c_{\max }\right)\right)_{\mid \mathbb{P}^{*}}$. 
Consider now $n>0$ and assume the property holds for $n-1$. Since $n>0$, then there exists a transition $t$ and a sequence $\sigma_{3}$ such that $\sigma_{2}=t . \sigma_{3}$. By definition of $\sigma_{2}$, we have $C_{\sigma_{1}} \in$ PASsed but $C_{\sigma_{1} . t} \notin$ PASsed. By Lemma 4 , since Waiting $=\emptyset$, there must exists some class $C_{\sigma^{\prime}}$ such that $C_{\sigma_{1} . t} \preccurlyeq C_{\sigma^{\prime}}$. From Lemma 3, sequence $\sigma_{3}$ is also firable from $C_{\sigma^{\prime}}$ and $C_{\sigma^{\prime} . \sigma_{3}}=\left(m, D^{\prime}\right)$, with $\operatorname{cost}\left(C_{\sigma^{\prime} . \sigma_{3}}\right) \leq \operatorname{cost}\left(C_{\sigma}\right) \leq c_{\max }$. By Lemma 1, there exists thus a run $\rho^{\prime}$ in $v(\mathcal{N})$, with sequence $\left(\rho^{\prime}\right)=\sigma^{\prime} . \sigma_{3}, \operatorname{lastm}\left(\rho^{\prime}\right) \in$ Goal and $\operatorname{cost}\left(\rho^{\prime}\right) \leq$ $c_{\max }$. Also, from Lemma 4 (item 1), we know that for all prefixes of $\sigma^{\prime}$, the corresponding state class is in PASSED, so the biggest suffix of $\sigma^{\prime} . \sigma_{3}$ as defined above in the induction hypothesis has length less or equal to $n-1$, and the induction hypothesis applies to $\rho^{\prime}$, which allows to conclude.

In particular, if the algorithm terminates, then the waiting list is empty and PolyRes is exactly the solution to the synthesis problem.

\section{$5 \quad$ Restricting to Integer Parameters}

Obviously, in general, (semi-)Algorithm 2 will not terminate, since the emptiness problem for the set it computes is undecidable.

To ensure termination, we can however follow the methodology of [15]: we require that parameters are bounded integers and, instead of just enumerating the possible parameter values, we propose a modification of the symbolic state computation to compute these integer parameters symbolically. For this we rely on the notion of integer hull.

We call integer valuation a $\mathbb{Z}$-valuation. Note that a $\mathbb{Z}$-valuation is also an $\mathbb{R}$-valuation, and given a set $D$ of $\mathbb{R}$-valuations, we denote by $\operatorname{lnts}(D)$ the set of integer valuations in $D$.

The convex hull of a set $D$ of valuations, denoted by $\operatorname{Conv}(D)$, is the intersection of all the convex sets of valuations that contain $D$.

The integer hull of a set $D$ of valuations, denoted by $\mathrm{IH}(D)$, is defined as the convex hull of the integer valuations in $D: \operatorname{lH}(D)=\operatorname{Conv}(\operatorname{Ints}(D))$.

For a state class $C=(m, D)$, we write $\mathrm{IH}(C)$ for $(m, \mathrm{IH}(D))$.

Before we see how our result can be adapted for the restriction to integer parameter valuations, and from there how we can enforce termination of the symbolic computations when parameters are assumed to be bounded, we need some results on the structure of the polyhedra representing firing domains of cost TPNs.

By the Minkowski-Weyl Theorem (see e.g. [20]), every convex polyhedron can be either described as a set of linear inequalities, as seen above, or by a set of generators. More precisely, for the latter: if $d$ is the dimension of polyhedron $P$, there exists $v_{1}, \ldots, v_{p}, r_{1}, \ldots, r_{s} \in \mathbb{R}^{d}$, such that for all points $x \in P$, there exists $\lambda_{1}, \ldots, \lambda_{p} \in \mathbb{R}, \mu_{1}, \ldots, \mu_{s} \in \mathbb{R}_{\geq 0}$ such that $\sum_{i} \lambda_{i}=1$ and $x=\sum_{i} \lambda_{i} v_{i}+\sum_{i} \mu_{i} r_{i}$. The $v_{i}$ 's are called the vertices of $P$ and the $r_{i}$ 's are the extremal rays of $P$. The latter correspond to the directions in which the polyhedron is infinite. In our case, they correspond to transitions with a (right-)infinite static interval, and possibly the cost. 
A classic property of vertices, which can also be used as a definition, is as follows: $\vec{v}$ is a vertex of $P$ iff for all non-null vectors $\overrightarrow{\vec{x}} \in \mathbb{R}^{d}$, either $\vec{v}+\vec{x} \notin P$ or $\vec{v}-\vec{x} \notin P$ (or both), + and - being understood component-wise.

Proposition 3. Let $\mathcal{N}$ be a (non-parametric) cost TPN and let $C=(m, D)$ be one of its state classes, then $D$ has integer vertices.

Proof. We have proved in [11] that the domain $D$ of a state class of a cost TPNs, with removed upper bounds on cost (so-called relaxed classes), can be partitioned into a union of simpler polyhedra $\bigcup_{i=1}^{n} D_{i}$ that have the following key properties: (1) by projecting the cost out we obtain a convex polyhedron $D_{i \mid \Theta}$ with integer vertices (actually a zone, as in $[17,9]$ ), and (2) these simpler polyhedra all have exactly one constraint on the cost variable, i.e., of the form $c \geq \ell(\vec{\theta})$, with integer coefficients. Note that the same result can be obtained, with the same technique, if we consider non-relaxed state classes, except that, we also have an upper bound on cost that is always greater or equal to the lower bound. We prove in Lemma 5 that each of these simpler polyhedra also has integer vertices. Since $D$ and each of the $D_{i}$ 's are convex and since $D=\bigcup_{i} D_{i}$, $D$ is equal to the convex hull of the vertices of the $D_{i}$ 's and therefore $D$ also has integer vertices.

Lemma 5. Let $D$ be a convex polyhedron on variables $\theta_{1}, \ldots, \theta_{n}, c$ such that the projection of $P$ on the $\theta$ variables has integer vertices, and there are two constraints on $c$ of the form $c \geq \ell\left(\theta_{1}, \ldots, \theta_{n}\right)$ and $c \leq \ell^{\prime}\left(\theta_{1}, \ldots, \theta_{n}\right)$, with $\ell$ and $\ell^{\prime}$ linear terms with integer coefficients, such that $\ell\left(\theta_{1}, \ldots, \theta_{n}\right) \leq \ell^{\prime}\left(\theta_{1}, \ldots, \theta_{n}\right)$, for all values of the $\theta_{i}$ 's.

Then, the vertices of $D$ are the points $\left(\theta_{1}, \ldots, \theta_{n}, \ell\left(\theta_{1}, \ldots, \theta_{n}\right)\right)$ and $\left(\theta_{1}, \ldots, \theta_{n}\right.$, $\left.\ell^{\prime}\left(\theta_{1}, \ldots, \theta_{n}\right)\right)$ such that $\left(\theta_{1}, \ldots, \theta_{n}\right)$ is a vertex of $D_{\mid \Theta}$, and they are integer points.

Proof. Recall here that we consider all constraints in $D$ to be non-strict so all polyhedra are topologically closed. The reasoning extends with no difficulty to non-necessarily-closed polyhedra by considering so-called closure points in addition to vertices [6].

Consider a non-vertex point $\vec{\theta}$ in $D_{\mid \Theta}$ and let $(\vec{\theta}, c)$ be a point of $D$. Then using the form of the unique cost constraint, we have $c \geq \ell(\vec{\theta})$. Now since $\vec{\theta}$ is not a vertex, there exists a vector $\vec{x}$ such that both $\vec{\theta}+\vec{x}$ and $\vec{\theta}-\vec{x}$ belong to $D_{\mid \Theta}$. Then, for sure, $(\vec{\theta}+\vec{x}, \ell(\vec{\theta}+\vec{x})) \in D$ and $(\vec{\theta}-\vec{x}, \ell(\vec{\theta}-\vec{x})) \in D$. And since $\ell$ is linear, $(\vec{\theta}+\vec{x}, \ell(\vec{\theta})+\ell(\vec{x}))) \in D$, i.e., $(\vec{\theta}, \ell(\vec{\theta}))+(\vec{x}, \ell(\vec{x})) \in D$. And similarly, $(\vec{\theta}, \ell(\vec{\theta}))-(\vec{x}, \ell(\vec{x})) \in D$. Using again the form of the unique cost constraint, and the fact that $c \geq \ell(\vec{\theta})$, we finally have $(\vec{\theta}, c)+(\vec{x}, \ell(\vec{x})) \in D$ and $(\vec{\theta}, c)-(\vec{x}, \ell(\vec{x})) \in D$, that is, $(\overrightarrow{\vec{\theta}}, c)$ is not a vertex of $D$.

By contraposition, any vertex of $D$ extends a vertex of $D_{\mid \Theta}$, and using a last time the form of the cost constraint, any vertex of $D$, is of the form $(\vec{\theta}, \ell(\vec{\theta}))$, with $\vec{\theta}$ a vertex of $D_{\mid \Theta}$ : suppose $(\vec{\theta}, c)$ is a vertex of $D$, with $c>\ell(\vec{\theta})$, then for 
$\vec{x}$ defined with $c-\ell(\vec{\theta})$ on the cost variable, and 0 on all other dimensions, we clearly have both $(\vec{\theta}, c)+\vec{x}$ and $(\vec{\theta}, c)-\vec{x}$ in $D$, which is a contradiction.

We conclude by remarking that, since $D_{\mid \Theta}$ has integer vertices, all the coordinates of $\vec{\theta}$ are integers, and since $\ell$ has integer coefficients then $\ell(\vec{\theta})$ is an integer.

We can deal with the upper bound defined by $\ell^{\prime}$ in exactly the same way.

From Proposition 3, we can prove the following lemma that will be very useful in the subsequent proofs.

Lemma 6. Let $(m, D)$ be a state class of a pcTPN and let $(\vec{\theta}, c, v)$ be a point in $D$.

If $v$ is an integer valuation, then $(\vec{\theta}, c, v) \in \mathrm{IH}(D)$.

Proof. Since $(\vec{\theta}, c, v) \in D$ then $(\vec{\theta}, c) \in v(D)$. By Proposition 3, $v(D)$ being the firing domain of a state class in a (non-parametric) cost TPN, it has integer vertices, and therefore $v(D)=\mathrm{IH}(v(D))$. Point $(\vec{\theta}, c)$ is therefore a convex combination of integer points in $v(D)$. Clearly, for all integer points $\left(\vec{\theta}^{\prime}, c^{\prime}\right)$ in $v(D)$, we have that $\left(\vec{\theta}^{\prime}, c^{\prime}, v\right)$ is an integer point of $D$. Since $D$ is convex, this implies that $(\vec{\theta}, c, v) \in \mathrm{IH}(D)$.

When we restrict ourselves to integer parameter but continue to work symbolically, we need to adjust the definitions of the firability of a transition from a class and of the cost of a class.

First, a transition $t_{f}$ is firable for integer parameter valuations from a class $(m, D)$, call this $\mathbb{N}^{\mathbb{P}}$-firable, if there exists an integer parameter valuation $v$ and a point $(\vec{\theta}, c, v)$ in $D$ such that for all transitions $t_{i} \in \operatorname{en}(m), \theta_{i} \geq \theta_{f}$.

Lemma 7. Let $C=(m, D)$ be a state class. Transition $t_{f} \in \operatorname{en}(m)$ is $\mathbb{N}^{\mathbb{P}}$-firable from $C$ if and only if it is firable (not necessarily $\mathbb{N}^{\mathbb{P}}$-firable) from $(m, \mathrm{IH}(D)$ ).

Proof. $\Leftarrow$ : trivial because $\mathrm{IH}(D) \subseteq D$.

$\Rightarrow$ : since $t_{f}$ is $\mathbb{N}^{\mathbb{P}}$-firable from $C$, there exists an integer parameter valuation $v$, and $(\vec{\theta}, c, v) \in D$ such that for all transitions $t_{i} \in \operatorname{en}(m), \theta_{i} \geq \theta_{f}$. And the result follows from Lemma 6 because $v$ is an integer valuation.

Second, the cost of a class $C=(m, D)$, for integer parameters, is $\operatorname{cost}_{\mathbb{N}}(C)=$ $\inf _{(\vec{\theta}, c, v) \in D, v \in \mathbb{N}^{\mathbb{P}}} c$.

Lemma 8 is a direct consequence of Lemma 6 :

Lemma 8. Let $(m, D)$ be a state class. We have: $\operatorname{cost}_{\mathbb{N}}((m, D))=\operatorname{cost}((m, \mathrm{IH}(D))$.

Lemma 9. If $v$ is an integer parameter valuation, then for all classes $C_{\sigma}=$ $(m, D),(\vec{\theta}, c, v) \in \mathrm{IH}(D)$ if and only if there exists a run $\rho$ in $v(\mathcal{N})$, and $I$ : en $(m) \rightarrow \mathcal{I}\left(\mathbb{Q}_{\geq 0}\right)$, such that sequence $(\rho)=\sigma,(m, I, c)=\operatorname{last}(\rho)$, and $\vec{\theta} \in I$. 
Proof. $\Rightarrow$ : if $(\vec{\theta}, c, v) \in \mathbf{I H}(D)$ then it is also in $D$ and the result follows from Lemma 1.

$\Leftarrow$ : by Lemma 1 , we know that there exists some $(\vec{\theta}, c, v) \in D$, and since $v$ is an integer valuation, by Lemma $6,(\vec{\theta}, c, v) \in \mathrm{IH}(D)$.

Lemma 10. Let $C_{\sigma_{1}}$ and $C_{\sigma_{2}}$ be two state classes such that $\mathrm{IH}\left(C_{\sigma_{1}}\right) \preccurlyeq \mathrm{IH}\left(C_{\sigma_{2}}\right)$. If a transition sequence $\sigma$ is $\mathbb{N}^{\mathbb{P}}$-firable from $C_{\sigma_{1}}$ it is also $\mathbb{N}^{\mathbb{P}}$-firable from $C_{\sigma_{2}}$ and $\operatorname{cost}_{\mathbb{N}}\left(C_{\sigma_{1} . \sigma}\right) \geq \operatorname{cost}_{\mathbb{N}}\left(C_{\sigma_{2} . \sigma}\right)$.

Proof. Let $C_{\sigma_{1}}=\left(m_{1}, D_{1}\right)$ and $C_{\sigma_{2}}=\left(m_{2}, D_{2}\right)$. From Definition 3, for any point $\left(\vec{\theta}, c_{1}, v\right) \in \mathrm{IH}\left(D_{1}\right)$, there exists a point $\left(\vec{\theta}, c_{2}, v\right) \in \mathrm{IH}\left(D_{2}\right)$ such that $c_{2} \leq c_{1}$. With Lemma 7 and Lemma 8, this implies that: (i) $\operatorname{cost}_{\mathbb{N}}\left(C_{\sigma_{1}}\right) \geq \operatorname{cost}_{\mathbb{N}}\left(C_{\sigma_{2}}\right)$; (ii) if transition $t$ is $\mathbb{N}^{\mathbb{P}}$-firable from $C_{\sigma_{1}}$, then it is $\mathbb{N}^{\mathbb{P}}$-firable from $C_{\sigma_{2}}$ and $\operatorname{Next}\left(C_{\sigma_{1}}, t\right) \preccurlyeq \operatorname{Next}\left(C_{\sigma_{2}}, t\right)$. And, as before, the result follows by a straightforward induction.

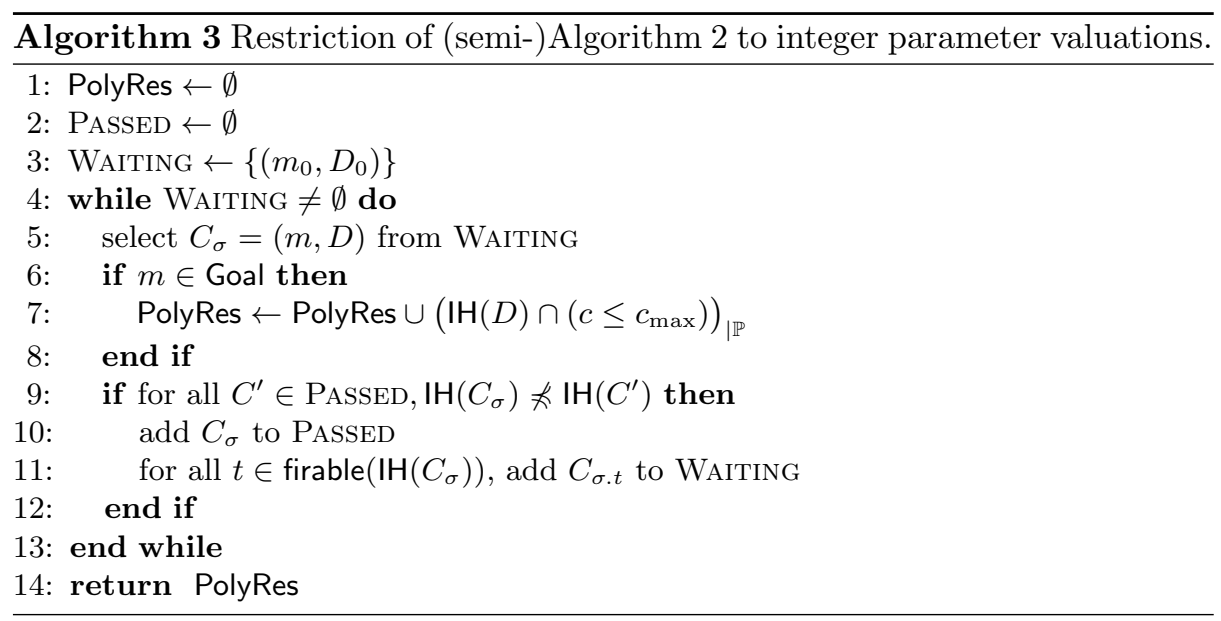

Using Lemma 9 instead of Lemma 1, and Lemma 10 instead of Lemma 3 in the proof of Proposition 2, we get the following proposition, stating the completeness and soundness of Algorithm 3.

Proposition 4. After any iteration of the while loop in Algorithm 3:

1. if $v \in$ PolyRes and $v$ is an integer parameter valuation then there exists a run $\rho$ in $v(\mathcal{N})$ such that $\operatorname{cost}(\rho) \leq c_{\max }$ and $\operatorname{lastm}(\rho) \in$ Goal.

2. if WAITING $=\emptyset$ then for all integer parameter valuations $v$ such that there exists a run $\rho$ in $v(\mathcal{N})$ such that $\operatorname{cost}(\rho) \leq c_{\max }$ and $\operatorname{lastm}(\rho) \in \operatorname{Goal}$, we have $v \in$ PolyRes. 
In Algorithm 3, we compute state classes as usual then handle them via their integer hulls. We can actually simply integrate integer hulls at the end of Algorithm 1 and use Algorithm 2 with this updated successor computation as proved by Lemma 11 .

Lemma 11. Let $(m, D)$ be a state class of a pcTPN $\mathcal{N}$, and $t$ a transition firable from $C$. Let $\left(m^{\prime}, D^{\prime}\right)=\operatorname{Next}((m, D), t)$ and $\left(m^{\prime \prime}, D^{\prime \prime}\right)=\operatorname{Next}((m, \operatorname{lH}(D)), t)$. Then $m^{\prime \prime}=m^{\prime}$ and $\mathrm{IH}\left(D^{\prime \prime}\right)=\mathrm{IH}\left(D^{\prime}\right)$.

Proof. The equality of markings is trivial so we focus on firing domains.

By definition of the integer hull, we have $\mathrm{IH}(D) \subseteq D$. Since the computation of the next class domain is non-decreasing with respect to inclusion, we then have $D^{\prime \prime} \subseteq D^{\prime}$. Taking the integer hull is also non-decreasing wrt. inclusion, so $\mathrm{IH}\left(D^{\prime \prime}\right) \subseteq \mathrm{IH}\left(D^{\prime}\right)$.

Consider now an integer point $\left(\overrightarrow{\theta^{\prime}}, c^{\prime}, v\right)$ in $D^{\prime}$. Then $\left(\overrightarrow{\theta^{\prime}}, c^{\prime}\right) \in v\left(D^{\prime}\right)$. Consider state class computations in the (non-parametric) cost TPN $v(\mathcal{N})$ : there exists

some point $(\vec{\theta}, c)$ in $v(D)$ such that $\left(m^{\prime}, \vec{\theta}^{\prime}, c^{\prime}\right) \in \operatorname{Next}((m,\{(\vec{\theta}, c)\}), t)$. Since $(\vec{\theta}, c, v)$ thus belongs to $D$ and since $v$ is an integer parameter valuation, by Lemma 6 , we have that $(\vec{\theta}, c, v) \in \mathrm{IH}(D)$. Thus $\left(\vec{\theta}^{\prime}, c^{\prime}, v\right) \in D^{\prime \prime}$ and since it is an integer point, it is in $\mathrm{IH}\left(D^{\prime \prime}\right)$.

\section{Termination of Algorithm 3}

We now consider that parameter valuations are bounded by some value $M_{1} \in \mathbb{N}$ (and that they still have integer values). We also assume that, for all integer parameter valuations, there exists $M_{2} \in \mathbb{Z}$ such that for all runs $\rho$ in $v(\mathcal{N})$, $\operatorname{cost}(\rho) \geq M_{2}$ : this allows us, as in [11,12], to keep Algorithm 3 simple by doing away with negative cost loop-checking. Finally, we assume the net itself is bounded: there exists $M_{3} \in \mathbb{N}$ such that for all reachable markings $m$, for all places $p, m(p) \leq M_{3}$.

To prove the termination of Algorithm 3 under these assumptions, we consider $\succcurlyeq$ the symmetric relation to $\preccurlyeq$, such that $x \succcurlyeq y$ iff $y \preccurlyeq x$. We prove that it is a well quasi-order (wqo), i.e., that for every infinite sequence of state classes, there exist $C$ and $C^{\prime}$ in the sequence, with $C$ strictly preceding $C^{\prime}$ such that $C \succcurlyeq C^{\prime}$. This implies that the exploration of children in Algorithm 3 will always eventually stop.

Proposition 5. Let $\mathcal{N}$ be a bounded pcTPN, with bounded integer parameters and such that the cost of all runs is uniformly lower-bounded for all integer parameter valuations.

Relation $\succcurlyeq$ is well-quasiorder on the set of state classes of $\mathcal{N}$.

Proof. Consider an infinite sequence $C_{0}, C_{1}, C_{2}, \ldots$ of state classes. Let $C_{i}=$ $\left(m_{i}, D_{i}\right)$.

From [11], we know that $\succcurlyeq$ is a wqo for the state classes of bounded (non parametric) cost TPNs. So for each integer parameter valuation $v$, and using a 
classic property of wqo we can extract a subsequence of $v\left(C_{0}\right), v\left(C_{1}\right), \ldots$ that is completely ordered by $\succcurlyeq$. And since, we have a finite number of such parameter valuations, we can extract an infinite subsequence $C_{i_{0}}, C_{i_{1}}, \ldots$ such that for all integer parameter valuations $v, v\left(C_{i_{0}}\right) \succcurlyeq v\left(C_{i_{1}}\right) \succcurlyeq \cdots$.

Let us consider two of those: $C_{i_{r}}$ and $C_{i_{s}}$, with $r<s$.

Since $\mathrm{IH}\left(D_{i_{s}}\right)$ has integer vertices, and for any integer parameter valuation, $v\left(C_{i_{r}}\right) \succcurlyeq v\left(C_{i_{s}}\right)$, which implies that $v\left(D_{i_{s}}\right) \subseteq v\left(D_{i_{r}}\right)$, then all the vertices of $D_{i_{s}}$ are also in $D_{i_{r}}$. Now assume that some extremal ray $\vec{r}$ of $D_{i_{s}}$ is not in $D_{i_{r}}$. Then starting from some vertex $\vec{x}$ of $D_{i_{r}}$, there must be some $\lambda \leq 0$ such that $\vec{x}+\lambda r \notin D_{i_{s}}$ and the same holds for any $\lambda^{\prime} \geq \lambda$ (by convexity). But since $r$ has rational coordinates for some value of $\lambda^{\prime}, \lambda^{\prime} r$ is an integer vector and so is $\vec{x}+\lambda^{\prime} r$, which contradicts the fact that $v\left(D_{i_{s}}\right) \subseteq v\left(D_{i_{r}}\right)$, for all integer parameter valuations $v$, and in particular $\left(\vec{x}+\lambda^{\prime} r\right)_{\mid \mathbb{P}}$. We can therefore conclude that $D_{i_{r}} \subseteq D_{i_{s}}$ and we now proceed to proving that $D_{i_{s}}$ is also "cheaper" than $D_{i_{r}}$.

We use another property of the vertices of convex polyhedra: vertices of a convex polyhedron of dimension $n$ defined by $m$ inequalities $\sum_{k=1}^{n} a_{k l} x_{k} \leq b_{l}$, for $j \in[1 . . m]$ are solutions of a system of $n$ linearly independent equations $\sum_{k=1}^{n} a_{k l} x_{k}=b_{l}$, with $l$ in a subset of size $n$ of $[1 . . m]$.

Now consider the polyhedron $D$ obtained from $\mathrm{IH}\left(D_{i_{r}}\right)$, with its cost variable $c$, by adding one variable $c^{\prime}$ constrained by the cost inequalities of $\mathrm{IH}\left(D_{i_{s}}\right)$. Clearly, since $c$ and $c^{\prime}$ are not constrained together, the vertices of $D$ are those of $\mathrm{IH}\left(D_{i_{r}}\right)$, extended with the corresponding minimal and maximal values of $c^{\prime}$, and symmetrically those of $\mathrm{IH}\left(D_{i_{s}}\right)$, extended with the corresponding minimal and maximal values of $c^{\prime}$. Since the inequalities constraining $c$ and $c^{\prime}$ have integer coefficients, and $\mathrm{IH}\left(D_{i_{s}}\right)$ and $\mathrm{IH}\left(D_{i_{r}}\right)$ have integer vertices, $D$ also has integer vertices.

For the $i$-th lower-bound inequality on $c$, and the $j$-th lower-bound inequality on $c^{\prime}$, we define $E_{i j}$ as $D$ in which we transform both constraints into equalities. Clearly, from the property above, this does not add any new vertex, but it may remove some. Second, by construction, we have $\bigcup_{i j} E_{i j}=$

$\left\{\left(\vec{\theta}, \min _{(\vec{\theta}, c, v) \in \mathrm{IH}\left(D_{i_{r}}\right)} c, \min _{(\vec{\theta}, c, v) \in \mathrm{H}\left(D_{i_{s}}\right)} c\right) \mid \vec{\theta} \in \mathrm{IH}\left(D_{r}\right)_{\mid \Theta}\right\}$. If we minimize $c-c^{\prime}$ over $E_{i}$, we know from the theory of linear programming that the minimum is obtained at a vertex of $E_{i j}$, and therefore, in particular, for an integer valuation $v$ of the parameters, and an integer vector $\vec{\theta}$ of $D_{i_{r}}$. Since we have $v\left(C_{i_{r}}\right) \succcurlyeq v\left(C_{i_{s}}\right)$, we then know that for these values of the theta variables and parameters, $c \leq c^{\prime}$. This means that this holds for the whole of $E_{i j}$, and finally that $C_{i_{r}} \succcurlyeq C_{i_{s}}$.

\section{Case Study}

We now consider a scheduling problem where some tasks include runnables, a key concept of the AUTomotive Open System ARchitecture (AUTOSAR), the open standard for designing the architecture of vehicle software [5]. Runnables represent the functional view of the system and are executed by the runtime of the software component [19]. For their execution they are mapped to tasks and 
a given runnable can be split across different tasks to introduce parallelism, for instance. In industrial practice, runnables that interact a lot are mapped to the same task, in particular when they perform functions with the same period.

In this example, we consider 3 non-preemptive, periodic tasks T1, T2 and T3, on which have already been mapped some runnables that interact together; we add another independent runnable whose code must be split between tasks T1 and T2:

- the period of task T1 is 100 time units; T1 includes a "fixed part", independent from the new runnable and whose execution lasts 22 t.u.;

- the period of T2 is 200 t.u.; T2 also has a fixed part lasting 28 t.u.;

- the period of T3 is 400 t.u.; its execution lasts 11 t.u.;

- the period of the runnable is 200 t.u.; its execution lasts 76 t.u.; parameter $a$ denotes the duration of the section that is executed in $\mathrm{T}^{3}$.

The processing unit consists of 2 cores $\mathrm{C} 0$ and $\mathrm{C} 1$; T3 can only execute on $\mathrm{C} 0$ whereas both T1 and T2 can execute on either core. When both cores are idle, the cost is null; when only one core is busy, the cost is equal to $2 /$ t.u.; when both cores are busy, the cost is equal to $3 /$ t.u. Any optimised strategy to divide the runnable over $\mathrm{T} 1$ and $\mathrm{T} 2$ and to allocate these tasks to $\mathrm{C} 0$ or $\mathrm{C} 1$ must therefore favour the cases where both cores are in the same state.

Figure 3 presents the model for this problem ${ }^{4}$. The associated cost function is: $2 *(C 0 \neq C 1)+3 * C 0 * C 1+1000 *(W 1 *(R 1 C 0+R 1 C 1)+W 2 *(R 2 C 0+R 2 C 1)+$ $W 3 * R 3 C 0$ ), where the name of a place (e.g. $R 1 C 0$ ) represents its marking ${ }^{5}$.

We limit the study of the system to the first 400 t.u., at the end of which $\mathrm{T} 1$ has been executed 4 times, T2 twice and T3 once. A preliminary analysis (not detailed here for the sake of concision) showed that the lowest cost is 466. By setting our maximal cost to this value, we then check the following property with our Romeo tool: $\mathrm{EF}$ four $==4$ and $\mathrm{two}==2$ and one $==1$ and cost $\leq 466$. The answer provided by Romeo is that the property is true iff $a \in[13,17]$. We then set $a$ to 17 ; Romeo yields the following timed trace, in which the notation T1@t1 means that transition T1 is fired at date t1: T1C0@61, T2C1@69, T1@100, end1_C0@100, T1C0@100, end1_C0@139, end2_C1@139, T1@200, T2@200, T2C0@261, T1C1@261, T1@300, end1_C1@300, T1C1@303, end2_C0@331, T3C0@331, end3_C0@342, end1_C1@342

From this trace, we obtain the Gantt chart in Figure 4 (above). Setting $a$ to 13 yields another timed trace, resulting in the Gantt chart in Figure 4 (below). In both cases, we can see that both cores are busy during 148 t.u. (and for 11 t.u., only one is idle), which confirms our analysis on the optimised strategy above.

${ }^{3}$ Every 200 t.u., since T1 is executed twice as often as T2, T1 is running during $(22+a) * 2=44+2 a$ t.u. whereas T2 is running during $28+(76-2 a)=104-2 a$ t.u.

4 To ensure a correct access to the cores, we could have added one place for each core and some arcs on each task to capture and release them but the resulting net would have been quite unreadable. Instead, we chose to add 2 integer variables $\mathrm{C} 0$ and $\mathrm{C} 1$ (both initialised to 0 ); a variable equal to 0 (resp. 1) obviously means the corresponding core is idle (resp. busy).

${ }^{5}$ The last term ensures that such cases where an instance of a task is activated while a previous one is running are heavily penalised. 

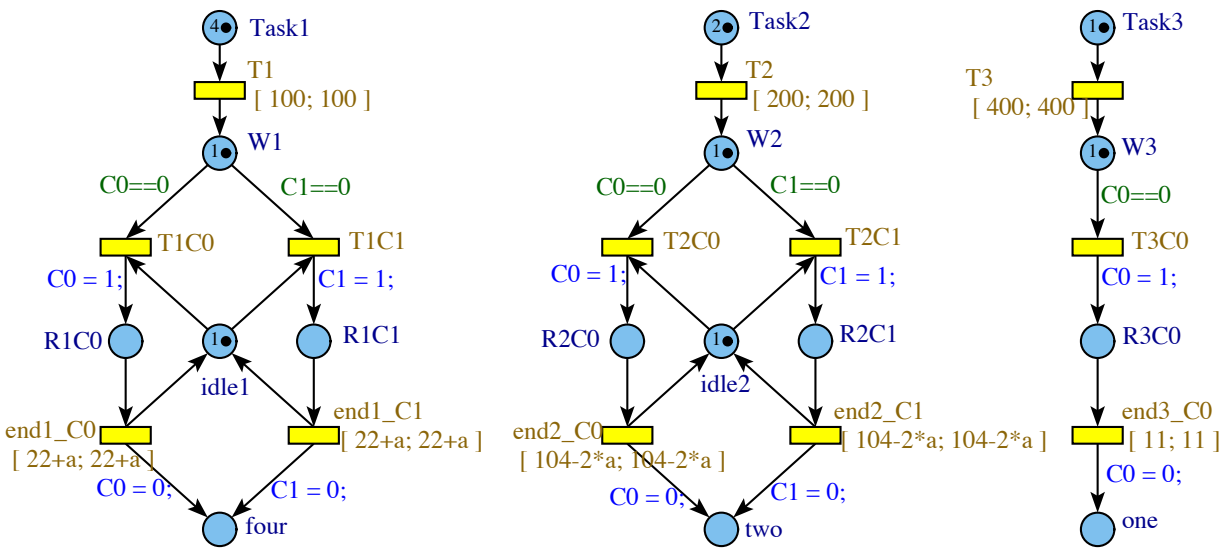

Fig. 3. Offline non preemptive scheduling problem
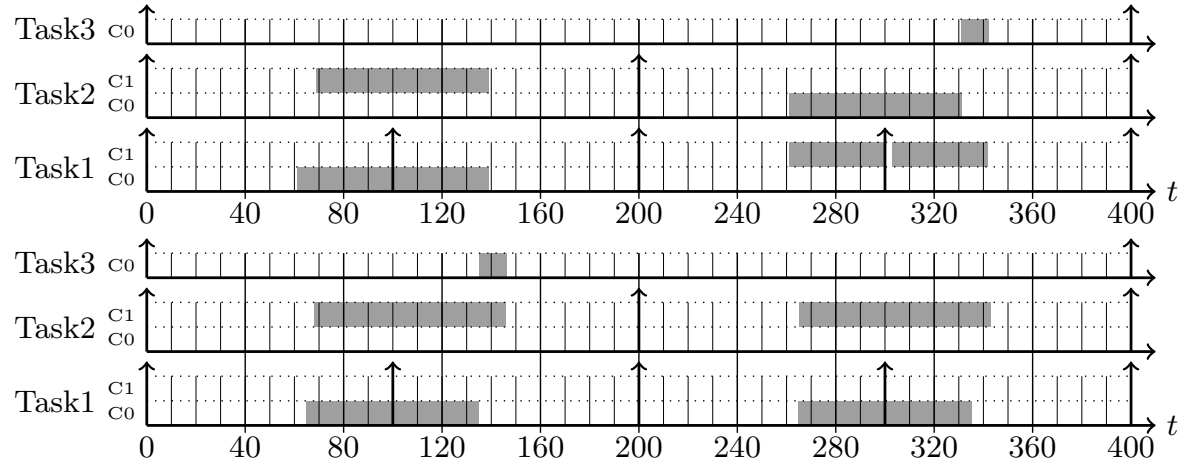

Fig. 4. Gantt charts for $a=17$ (above) and $a=13$ (below)

\section{Conclusion}

We have proposed a new Petri net-based formalism with parametric timing and cost features, thus merging two classic lines of work. For this formalism, we define an existential problem and a synthesis problem for parametric reachability within a bounded cost. We prove that the former is undecidable but we nonetheless give and prove a symbolic semi-algorithm for the latter. We finally propose a variant of the synthesis algorithm suitable for integer parameter valuations and prove its termination when those parameter valuations are bounded, and some other classic assumptions. This symbolic algorithm avoids the explicit enumeration of all possible parameter valuations. It is implemented in our tool Romeo and we have reported on a case-study addressing a scheduling problem, and inspired by the AUTOSAR standard.

Further work includes computing the optimal cost as a function of parameters and investigating the case of costs (discrete and rates) as parameters. 


\section{References}

1. P. A. Abdulla and R. Mayr. Priced timed Petri nets. Logical Methods in Computer Science, 9(4), 2013.

2. R. Alur and D. Dill. A Theory of Timed Automata. Theoretical Computer Science, 126(2):183-235, 1994.

3. R. Alur, T. A. Henzinger, and M. Y. Vardi. Parametric real-time reasoning. In ACM Symposium on Theory of Computing, pages 592-601, 1993.

4. R. Alur, S. L. Torre, and G. J. Pappas. Optimal paths in weighted timed automata. Theoretical Computer Science, 318(3):297 - 322, 2004.

5. AUTOSAR. Specification of RTE Software. Technical Report 4.4.0, october 2018.

6. R. Bagnara, P. Hill, and E. Zaffanella. Not necessarily closed polyhedra and the double description method. Formal Aspects of Computing, 17:222-257, 2005.

7. G. Behrmann, A. Fehnker, T. Hune, K. Larsen, P. Pettersson, J. Romijn, and F. Vaandrager. Minimum-cost reachability for priced timed automata. In HSCC 2001 Rome, Italy, pages 147-161. Springer, 2001.

8. G. Behrmann, K. G. Larsen, and J. I. Rasmussen. Optimal scheduling using priced timed automata. SIGMETRICS Perform. Eval. Rev., 32(4):34-40, Mar. 2005.

9. B. Berthomieu and M. Diaz. Modeling and verification of time dependent systems using time Petri nets. IEEE trans. on soft. eng., 17(3):259-273, 1991.

10. B. Berthomieu and M. Menasche. An enumerative approach for analyzing time petri nets. In IFIP, pages 41-46. Elsevier Science Publishers, 1983.

11. H. Boucheneb, D. Lime, B. Parquier, O. H. Roux, and C. Seidner. Optimal reachability in cost time Petri nets. In FORMATS'17, Berlin, Germany, LNCS, 2017.

12. P. Bouyer, M. Colange, and N. Markey. Symbolic optimal reachability in weighted timed automata. In $C A V^{\prime} 16$, volume 9779 of $L N C S$, Toronto, Canada, 2016.

13. T. Hune, J. Romijn, M. Stoelinga, and F. Vaandrager. Linear parametric model checking of timed automata. Journal of Logic and Algebraic Programming, 5253:183-220, 2002.

14. A. Jovanović. Parametric Verification of Timed Systems. PhD thesis, École Centrale Nantes, Nantes, France, 2013.

15. A. Jovanović, D. Lime, and O. H. Roux. Integer parameter synthesis for real-time systems. IEEE Transactions on Software Engineering (TSE), 41(5):445-461, 2015.

16. K. Larsen, G. Behrmann, E. Brinksma, A. Fehnker, T. Hune, P. Pettersson, and J. Romijn. As cheap as possible: Efficient cost-optimal reachability for priced timed automata. In $C A V^{\prime} 01$, volume 2102 of $L N C S$, pages 493-505, 2001.

17. K. G. Larsen, P. Pettersson, and W. Yi. Model-Checking for Real-Time Systems. In Fundamentals of Computation Theory, volume 965 of LNCS, pages 62-88, 1995.

18. M. Minsky. Computation: Finite and Infinite Machines. Prentice Hall, 1967.

19. N. Naumann. AUTOSAR runtime environment and virtual function bus. Technical report, Hasso-Plattner-Institut, 2009.

20. A. Schrijver. Theory of linear and integer programming. John Wiley \& Sons, Inc., New York, NY, USA, 1986.

21. L.-M. Traonouez, D. Lime, and O. H. Roux. Parametric model-checking of stopwatch Petri nets. Journal of Universal Computer Science, 15(17):3273-3304, 2009. 